“ (C) 2016 IEEE. Personal use of this material is permitted. Permission from IEEE must be obtained for all other uses, in any current or future media, including

reprinting/republishing this material for advertising or promotional purposes, creating new collective works, for resale or redistribution to servers or lists, or reuse of any copyrighted component of this work in other works." 


\title{
Techniques for RF Localization of Wireless Capsule Endoscopy
}

\author{
Ananda Sanagavarapu Mohan ${ }^{1} \quad$ Anantasri Boddupalli ${ }^{2}$ \\ Farhad Gozasht $^{2} \quad$ Steve S. H. Ling ${ }^{1}$ \\ Md. Delwar Hossain ${ }^{1}$
}

\begin{abstract}
Location estimation of a wireless capsule endoscope at $400 \mathrm{MHz}$ MICS band is implemented here using both RSSI and TOA-based techniques and their performance investigated. To improve the RSSI-based location estimation, a maximum likelihood (ML) estimation method is employed. For the TOA-based localization, FDTD coupled with continuous wavelet transform (CWT) is used to estimate the time of arrival and localization is performed using multilateration. The performances of the proposed localization algorithms are evaluated using a computational heterogeneous biological tissue phantom in the $402 \mathrm{MHz}-405 \mathrm{MHz}$ MICS band. Our investigations reveal that the accuracy obtained by TOA based method is superior to RSSI based estimates. It has been observed that the ML method substantially improves the accuracy of the RSSI-based location estimation.
\end{abstract}

\section{INTRODUCTION}

Bowel cancer is the most common internal cancer in Australia as it affects about 1 in 20 people with mortality rates second only to lung cancer. For prevention, early diagnosis using screening tests and continued surveillance of those in high risk groups are recommended. Wireless Capsule Endoscopy (WCE) is gaining acceptability for detecting bleeding in hidden areas in the small bowel. The WCE involves swallowing a small capsule equipped with a radio frequency (RF) antenna and transmitter, battery, LED light source and a tiny color camera. The RF transmitter uses the antenna to transmit the captured images wirelessly to the externally placed receivers, usually worn in a harness around the waist [1]. These images can then be used for diagnosing anomalies such as bleeding in the GI track or in the small bowel, for tracking small bowel tumors, etc. amongst many others. However, it is vital that the capsule position be estimated as precisely as possible, so as to isolate regions of abnormalities from the captured images. However, the biological tissue medium inside the human body is complex, lossy and heterogeneous, and hence it is still a challenge to accurately localize and reconstruct the movement of a WCE inside the GI tract.

Generally, the localization methods for a WCE can be categorized as based on use of low frequency magnetic fields or RF electromagnetic (EM) fields [2]. Even though the human body is non-magnetic and allows the magnetic field to pass through without attenuation, the presence of any conducting materials in its surroundings can perturb the fields, thus making the magnetic localization process difficult to perform accurately. Hence, RF localization techniques are preferable. The RF localization can be further categorized into those that use the narrowband or wideband/ultra wideband techniques. The narrowband RF localization techniques either use the signal strengths $[4,7]$ or angles of arrival of received signals [8]. The wideband and ultra wideband RF signals mainly make use of time delays such as time of arrival (TOA) between various transceivers and use triangulation to localize the sources [9]. Time based techniques require strict time synchronization and a sharp pulse with high bandwidth for obtaining the precision localization but can provide highly accurate localization $[8,9]$.

The localization using the received signal strength indication (RSSI) is a commonly employed due to its simplicity. RSSI based localization technique exploits the attenuation of the received signal strength with distance from the source [4,7]. The location estimation depends on the path loss of the propagation channel model, which is characterized by several parameters including the distance between the in-body transmitter (WCE) and the receivers located on or outside the body. In order to improve the performance of the RSSI-based localization, several methods such as the maximum likelihood (ML) estimation have been proposed [7]. All these methods require an appropriate channel model of the GI tract.

In this paper, we present wireless capsule localization using both RSSI -based technique as well as TOA based methods and compare their relative accuracies. The GI tract has been modeled using 3-D oval shaped, finite-sized, multilayered lossy dielectric cylinder. For RSSI-based methods, we obtain data on received powers at $400 \mathrm{MHz}$ medical implant communication service (MICS) band using a frequency domain solver [5] and use ML method to estimate the location [7]. For TOA based localization, we use FDTD simulations coupled with the continuous wavelet transform (CWT) to estimate time of arrivals of the signals at all receivers and use triangulation for localization. We compare the localization results obtained by both the above methods.

\section{NUMERICAL PHANTOM OF THE GI TRACT}

To localize the wireless capsule as it moves through the small intestine, a numerical 3-D small intestine

\footnotetext{
${ }^{1}$ Center for Health Technologies, Faculty of Engineering and Information Technology, University of Technology Sydney, NSW, Australia, e-mail: \{ Ananda.Sanagavarapu, Md.Hossain, Steve.Ling\}@uts.edu.au.

${ }^{2}$ Center for Health Technologies, Faculty of Engineering and Information Technology, University of Technology Sydney, NSW, Australia, e-mail: \{Anantasri.Boddupalli and Farhad.Gozasht\}@student.uts.edu.au
} 
phantom (SIP) having an elliptical shape is employed, as shown in Figure 1. The height $\left(H_{S I P}\right)$ of the phantom is $200 \mathrm{~mm}$, with the elliptical crosssection having a major axis $\left(M a_{S I P}\right)$ dimension of $170 \mathrm{~mm}$ and a minor axis $\left(M i_{S I P}\right)$ dimension of $90 \mathrm{~mm}$. In order to make the phantom more realistic, another cylindrical structure is added to represent the colon $(C P)$ and both these phantoms are immersed in blood $(B P)$. A small dipole antenna representing the wireless capsule transmitter is placed inside the small intestine phantom. 72 ideal receiver antennas are placed outside the body, in 6 equidistant rings of 12 antennas and at a distance of $5 \mathrm{~mm}$ from the skin. The receivers are placed in such a way so as to cover the entire vertical dimension of the phantom. The parameters used are listed in Table 1. The dielectric constants and conductivities used are listed in Table 2 [6].

For TOA estimation, we used the same phantom but employed FDTD simulation. A differentiated Gaussian pulse is used for excitation, with $3 \mathrm{~dB}$ bandwidth in $300 \mathrm{MHz}-1 \mathrm{GHz}$. The wireless capsule is also modeled using a small dipole antenna and the FDTD grid size is set to $2.5 \mathrm{~mm}$ which is uniform in all directions. The computational domain is truncated using PML boundary.

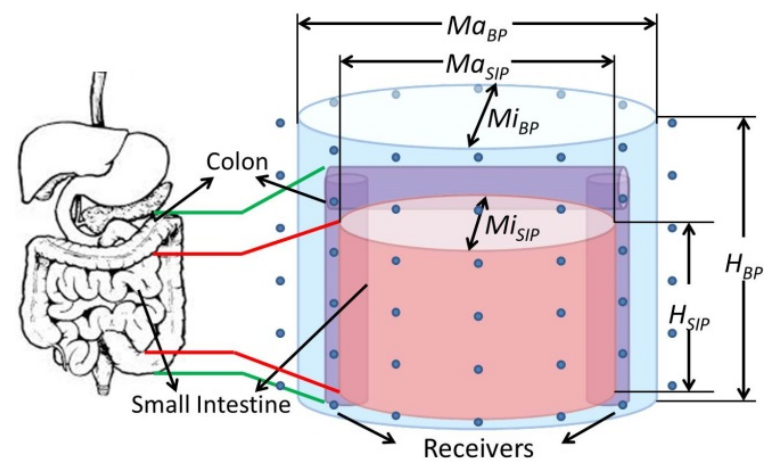

Figure 1: Numerical phantom used for investigation.

\begin{tabular}{|c|c|c|c|c|c|}
\hline $\boldsymbol{H}_{\boldsymbol{S I P}}$ & $\boldsymbol{M a}_{\boldsymbol{S I P}}$ & $\boldsymbol{M i}_{\boldsymbol{S I P}}$ & $\boldsymbol{H}_{\boldsymbol{B P}}$ & $\boldsymbol{M a}_{\boldsymbol{B P}}$ & $\boldsymbol{M i}_{\boldsymbol{B P}}$ \\
\hline 200 & 170 & 90 & 260 & 280 & 170 \\
\hline
\end{tabular}

Table 1: Phantom dimensions in $\mathrm{mm}$.

\begin{tabular}{|c|c|c|}
\hline Layer & $\boldsymbol{\epsilon}_{\boldsymbol{r}}$ & $\boldsymbol{\sigma}_{\boldsymbol{r}}[\mathbf{S} / \mathbf{m}]$ \\
\hline Blood & 66.045 & 1.904 \\
\hline Small Intestine & 64.144 & 1.351 \\
\hline Colon & 62.529 & 0.859 \\
\hline
\end{tabular}

Table 2: Dielectric constants and conductivities used for the different layers of the phantom.

\section{RSSI-BASED LOCALIZATION}

The RSSI provides the attenuation that the RF signal undergoes with the distance traversed and is usually modeled with a log-normal channel model that can be expressed as [3]

$$
\begin{aligned}
& \operatorname{RSSI}(d)_{[d B]} \\
& \quad=R S S I_{0[d B]}-10 \cdot n \cdot \log _{10}\left(d / d_{0}\right)+X_{[d B]}
\end{aligned}
$$

where, $R S S I_{0[d B]}$ is the reference power received at the distance $d_{0}, n$ is the path loss exponent, $d\left(\geq d_{0}\right)$ is the distance between the transmitter and receiver and $X$ is the zero-mean log-normally distributed Gaussian random variable representing the shadow fading. Figure 2 shows a scatter plot of the RSSI obtained by simulation [5] using the numerical phantom shown in Figure 1. A straight line is fitted to the computed RSSI data using least squares technique, to be used for path loss exponent prediction.

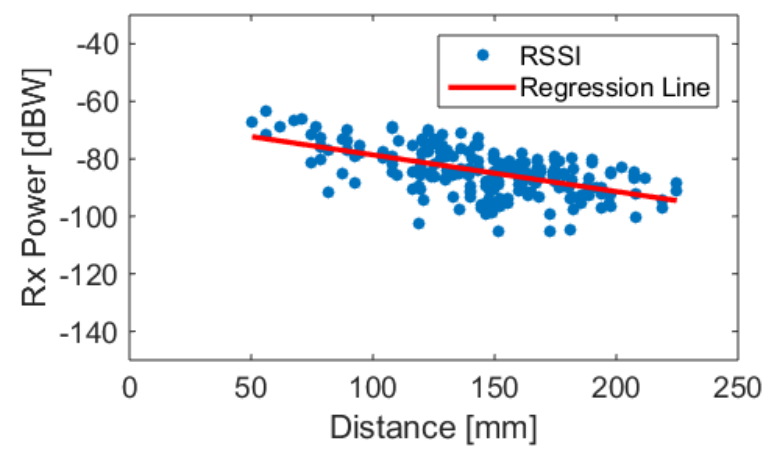

Figure 2: RSSI scatter plot.

The path loss exponent $(n)$ is estimated using the minimum mean square error (MMSE) minimization, given by

$$
J(n)=\sum_{i=1}^{K}\left(R S S I_{i}-\widehat{R S S I}_{l}\right)^{2}
$$

where, $i=1, \ldots, K$ denote the no. of receivers, $R S S I_{i}$ are obtained using FEKO[5] employing the numerical phantom shown in Figurel and $\widehat{R S S I}_{\imath}$ are the calculated data using equation (1). The standard deviation $(\sigma d B)$ of the lognormal shadowing variable has also been obtained from the MMSE procedure. The probability distribution function of the RSSIs in the GI tract channel at MICS band is given by [7]

$$
\begin{aligned}
& p\left(R S S I_{d B} \mid d\right) \\
& =\frac{1}{R S S I \cdot \sigma \cdot \sqrt{2 \pi}} \exp \left\{-\frac{[R S S I-\mu(d)]^{2}}{2 \sigma^{2}}\right\} \\
& \quad \mu(d)=\log (\overline{R S S I})=\log \left(R S S I_{0} \cdot d^{-n}\right)
\end{aligned}
$$




\subsection{Localization using ML algorithm}

Let the position of the wireless capsule given by

$$
P=\left[\begin{array}{lll}
x_{s} & y_{s} & z_{s}
\end{array}\right]^{T}
$$

and the fixed receiver locations are given as

$$
R_{i}=\left[x_{i r}, y_{i r}, z_{i r}\right]^{T}
$$

where, $i=1, \ldots, K$ represents the receivers. The wireless capsule is assumed to transmit $j(=1, \ldots, Y)$ packets to the receivers and the RSSI is measured for each packet that is received. Now, the distance between the capsule endoscope and the $i$-th receiver is given by

$$
d_{i}=\sqrt{\left(x_{s}-x_{i r}\right)^{2}+\left(y_{s}-y_{i r}\right)^{2}+\left(z_{s}-z_{i r}\right)^{2}}
$$

The log-likelihood function on $P$ can be written as [7]

$$
\begin{aligned}
& \mathcal{L}(P)=\ln \left[\prod_{j=1}^{Y} \prod_{i=1}^{K} p\left(R S S I_{i j} \mid d_{i}\right)\right] \\
& =\sum_{j=1}^{Y} \sum_{i=1}^{K}-\ln \left(R S S I_{i, j} \cdot \sigma \cdot \sqrt{2 \pi}\right)-\frac{\left[R S S I_{i, j}-\mu\left(d_{i}\right)\right]^{2}}{2 \sigma^{2}}
\end{aligned}
$$

The ML estimated position $\hat{P}_{M L}$ of the wireless capsule is found by maximizing the log-likelihood function given in equation (8). This can be achieved by

$$
\left.\frac{\partial \mathcal{L}(P)}{\partial C}\right|_{P=\hat{P}_{M L}}=0
$$

\section{TOA-BASED LOCALIZATION TECHNIQUE}

We employ the same numerical phantom shown in Figure 1 and use FDTD to obtain fields received and estimate their times of arrival at receivers using continuous wavelet transform (CWT). CWT compares a test signal with a time-scaled reference signal called wavelet. CWT is very effective in detecting abrupt changes in the signal [10]. Since biological tissue medium is lossy and dispersive in nature, CWT is well suited for TOA estimation in complex biological medium. We have used complex Gaussian wavelet function defined as

$$
\psi_{n}(t)=C_{n} \frac{d^{n}}{d t^{n}}\left(e^{-j t} e^{-t^{2}}\right)
$$

where, $C_{n}$ is a normalization constant chosen as $\left\|\psi_{n}\right\|^{2}=1$. CWT is defined for scaling parameter, $\alpha$ and position parameter, $\tau$, as

$$
\begin{aligned}
W(\alpha, \tau)=s(\tau) * & {\left[\frac{1}{\sqrt{\alpha}} \psi_{n}^{*}\left(\frac{\tau}{\alpha}\right)\right] } \\
& =\int_{-\infty}^{\infty} s(t) \psi_{n}^{*}\left(\frac{t-\tau}{\alpha}\right) d t
\end{aligned}
$$

As a result, $W_{s}(\alpha, \tau)$ is obtained as a $2 \mathrm{D}$ function of various scaling and time delay parameters. TOA is then estimated as

$$
\left(\alpha_{m}, \tau_{m}\right)=\max _{\alpha, \tau}\{|W(\alpha, \tau)|\}
$$

Once TOAs are obtained, the distance estimates between the capsule and the $n$-th receiver is given by

$$
d_{n}=\frac{c \tau_{n}}{\sqrt{\epsilon_{\text {med }}}}
$$

Here, $\tau_{n}$ is the TOA at the $n$-th receiver, $c$ is the free space propagation speed and $\epsilon_{\text {med }}$ is the dielectric constant of the medium. Since we have many receivers available, the localization can be obtained using multilateration $[9,11]$.

\section{EVALUATION OF LOCALIZATION PERFORMANCE}

RSSI method: A test scenario is setup in accordance with the channel model parameters that were estimated in the previous section. Next, an experimental environment is setup representing a human torso in the shape of a cuboid of dimensions $\mathrm{A}=0.3 \mathrm{~m}$ (width) $\times M=0.2 \mathrm{~m}$ (depth) $\times N=0.5 \mathrm{~m}$ (height) [12]. The wireless capsule is placed randomly at different locations within the cuboid and 8 receivers are placed at all its vertices. The channel model parameters used are $R S S I_{0}=-79.4 \mathrm{~dB}, n=$ 3.53 and standard deviation of log-normal shadowing parameter, $\sigma=5.475 \mathrm{~dB}$. Figure 4 shows RMSE of the localization performance versus number packets received. It can be seen from Figure 4, that the capsule location is estimated fairly accurately.

TOA method: Now we consider the localization results obtained by TOA-based multilateration technique which are plotted in Figure 6. The actual capsule location is indicated by the blue crossmarkers and the estimated capsule locations are indicted by the red circle-markers. It can be observed that TOA based multilateration technique can quite accurately estimate the capsule location, as expected. 


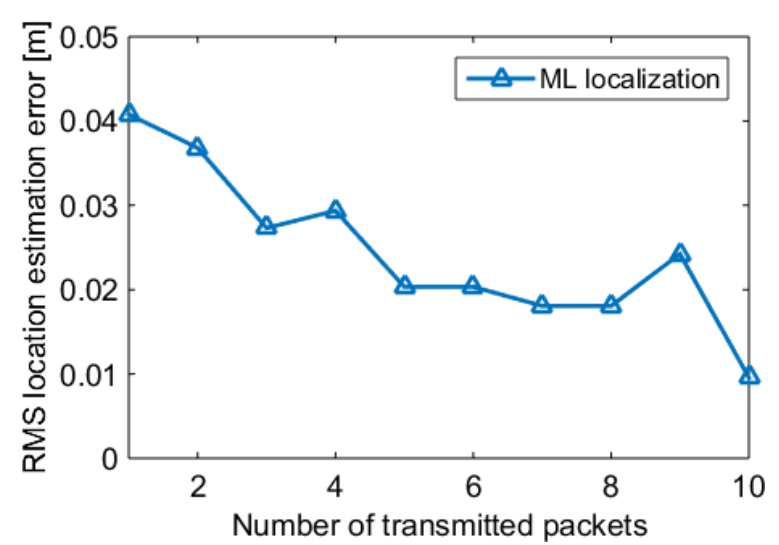

Figure 5: RMS error of localization estimation.

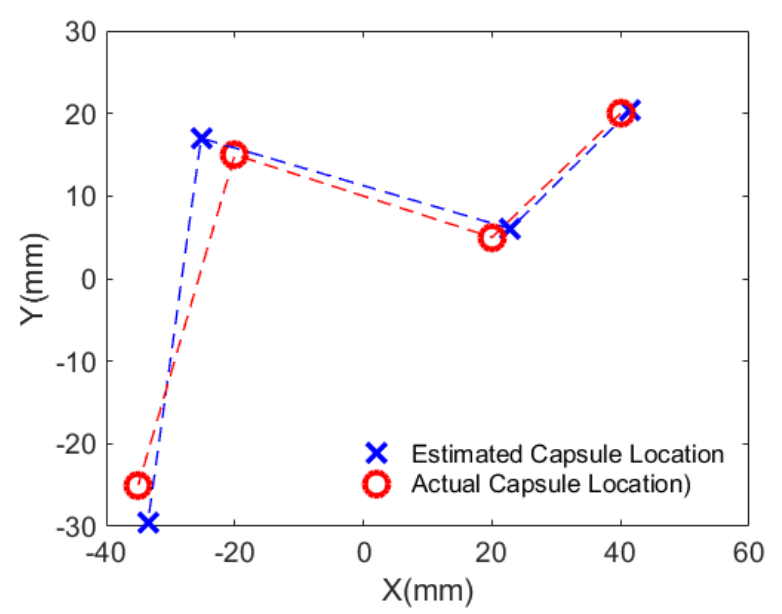

Figure 6: TOA-based capsule localization.

\section{CONCLUSIONS}

Localization techniques of wireless capsule inside the small intestine are presented. A heterogeneous numerical phantom has been constructed for modeling the RF propagation through the body tissues. For RSSI based localization, the statistical channel model is derived in the MICS $400 \mathrm{MHz}$ band based on the $\mathrm{FEKO}^{\circledR}$ simulations on a heterogeneous phantom model. Maximum Likelihood (ML) based estimation Is employed to obtain location estimation from RSSI data.

A TOA-based multilateration technique for localization is also implemented for the same heterogeneous phantom of small intestine and location estimation with higher accuracy is obtained. In future, we will consider a hybrid approach that incorporates both the RSSI and TOA techniques.

\section{References}

[1] G. Ciuti, A. Menciassi and P. Dario, "Capsule Endoscopy: From Current Achievements to Open Challenges", IEEE Review in Biomedical Engineering, Vol. 4, No.1, pp. 59-72, Oct. 2011.

[2] T.D. Than, G. Alici, H. Zhou, and Weihua Li, "A Review of Localization Systems for Robotic Endoscopic Capusles", IEEE Transactions on Biomedical Engineering., Vol. 59, No. 9, pp. 2387-2399, Sept. 2012.

[3] Y. Ye, K. Pahlavan, G. Bao, P. Swar, and K. Ghaboosi, "Comparitive Performance Evaluation of RF Localization for Wireless Capsule Endoscopy Applications", Int. Journal Wireless Inf. Net., Vol. 21, pp. 208-222, 2014.

[4] R. Zemek, D. Anzai, S. Hara, K. Yanagihara, and K. Kitayama, "RSSI-based Localization Without a Prior Knowledge of Channel Model Parameters", Int. Journal Wireless Inf. Net., Vol. 15, pp. 128-136, 2008.

[5] FEKO $^{\circledR}$ - EM Simulation Software (v7.1), Altair Engineering (https://www.feko.info).

[6] Dielectric Properties of Body Tissues (IFAC), available (http://niremf.ifac.cnr.it/tissprop/).

[7] D. Anzai, S. Aoyama and J. Wang, "Performance evaluation on RSSI-based localization for capsule endoscopy systems with $400 \mathrm{MHz}$ MICS band signals", IEICE Trans. Commun., vol.E95-B, no.10, pp.3081-3087, Oct. 2012.

[8] S. T. Goh, S. A. Zekavat, and K. Pahlavan, "DOA-Based Endoscopy Capsule Localization and Orientation Estimation via Unscented Kalman Filter", IEEE Sensors Journal, Vol. 14, no. 11, pp.3819-3829, Nov. 2014.

[9] U. I. Khan, K. Pahlavan, and S. Makarov, "Comparison of TOA and RSS based techniques for RF localization inside human tissue," in Proc. Annu. Int. Conf. IEEE Eng. Med. Biol. Soc., Boston, MA, USA, Aug./Sep. 2011, pp. 56025607.

[10] J. Sadowsky, "Investigation of Signal Characteristics Using the Continuous Wavelet Transform', Johns Hopkins APL Technical Digest, Vol. 17, no. 3, pp.258-269, 1996.

[11] F. Izquierdo, M. Ciurana, F.Barcelo, J. Paradells and E. Zola, "Performance evaluation of a TOAbased trilateration method to locate terminals in WLAN", Proc. 2006 1st International Symposium on Wireless Pervasive Computing, pp.1-6, 16-18 Jan.2016.

[12] I.Vitas, D.Zrno, D.Simunic and R.Prasad, "Innovative RF localization for wireless video capsule Endoscopy", Proc. ITU Kaleidoscope Academic Conference: Living in a Converged World-Impossible without Standards?, pp.221228, 3-5 June 2014. 